\title{
Micro- and Nanopatterned Silk Substrates for Antifouling Applications
}

\author{
G. Tullii, S. Donini, C. Bossio, F. Lodola, M. Pasini, E. Parisini, F. Galeotti, and M. R. Antognazza*
}

Cite This: ACS Appl. Mater. Interfaces 2020, 12, 5437-5446

Read Online

ABSTRACT: A major problem of current biomedical implants is the bacterial colonization and subsequent biofilm formation, which seriously affects their functioning and can lead to serious post-surgical complications. Intensive efforts have been directed toward the development of novel technologies that can prevent bacterial colonization while requiring minimal antibiotics doses. To this end, biocompatible materials with intrinsic antifouling capabilities are in high demand. Silk fibroin, widely employed in biotechnology, represents an interesting candidate. Here, we employ a soft-lithography approach to realize micro- and nanostructured silk fibroin substrates, with different geometries. We show that patterned silk film substrates support mammal cells (HEK-293) adhesion and proliferation, and at the same time, they intrinsically display remarkable antifouling properties. We employ Escherichia coli as

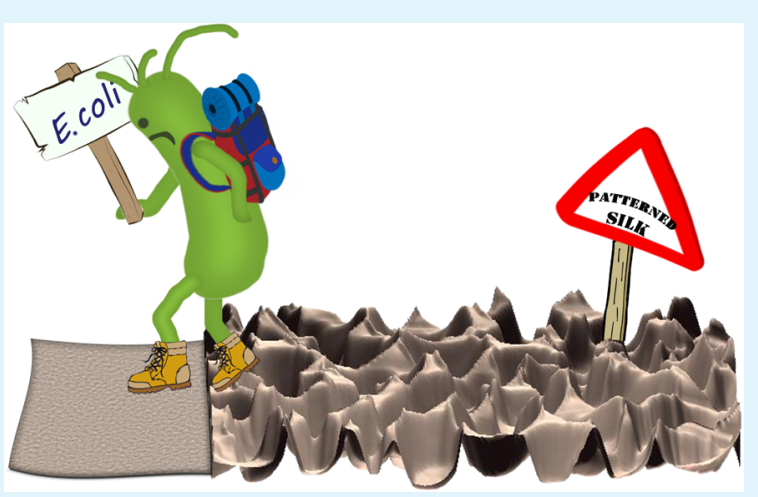
representative Gram-negative bacteria, and we observe an up to $66 \%$ decrease in the number of bacteria that adhere to patterned silk surfaces as compared to control, flat silk samples. The mechanism leading to the inhibition of biofilm formation critically depends on the microstructure geometry, involving both a steric and a hydrophobic effect. We also couple silk fibroin patterned films to a biocompatible, optically responsive organic semiconductor, and we verify that the antifouling properties are very well preserved. The technology described here is of interest for the next generation of biomedical implants, involving the use of materials with enhanced antibacterial capability, easy processability, high biocompatibility, and prompt availability for coupling with photoimaging and photodetection techniques.

KEYWORDS: silk fibroin, antifouling, conjugated polymer, microstructured surface, E. coli, breath figures, biofilm inhibition

\section{INTRODUCTION}

In recent years, silk fibroin has been eliciting ever-increasing interest in the biotechnology field. Several applications have been reported, spanning tissue engineering ${ }^{1,2}$ to regenerative medicine, $^{3-9}$ drug delivery ${ }^{10-12}$ to biophotonics, ${ }^{13-20}$ and implantable devices ${ }^{21-25}$ to the last frontiers in bioengineering and the realization of biodegradable, bioresorbable, and edible electronic devices. ${ }^{26-30}$ The main reason behind such a success story is the peculiar advantages offered by silk, in terms of outstanding mechanical properties, chemical-physical versatility due to its polymorphic character, thermal and environmental stability, suitability to several processing techniques (easily allowing for covering dimensions from the nano- to the macroscale), endless opportunities of functionalization with biomolecules and drugs, and excellent cytocompatibility. Overall, silk is widely recognized as a highly promising material platform, with global impact in the biomedical, biophotonics, and bioelectronics fields. ${ }^{31}$

The development of silk-based materials for implantable biotechnological devices has fostered investigation of the benefits of silk functionalization with antimicrobial agents. ${ }^{32-35}$ In fact, the main, hardly addressable risk leading to failure of medical implants is represented by microbial contamination, which causes severe device-associated infections. ${ }^{36}$ Bacteria preferentially adhere and proliferate on the implant surface, producing a biofilm, i.e., a layer of aggregated bacteria embedded into a matrix composed of extracellular polymeric substances such as proteins, DNA, and polysaccharides. ${ }^{37}$ The presence of the biofilm confers to the microorganisms a much higher resistance than the unattached bacteria, requiring 5005000 times higher doses of antibiotics. ${ }^{37,38}$ Some literature reports have addressed this problem by taking advantage of the chemical versatility of silk and by functionalizing the silk-based implant with antimicrobial agents. Unfortunately, this approach, albeit capable of substantially reducing bacterial contamination, has a serious drawback, since the extensive use of antibiotics inevitably leads to an enhanced bacterial

Received: October 7, 2019

Accepted: January 9, 2020

Published: January 9, 2020 
resistance toward the common antibiotic agents, thus establishing a vicious cycle.

A solution consists of hindering the formation of the biofilm (antifouling) by properly engineering implanted materials and devices. ${ }^{39}$ It has been widely reported that microbial adhesion can be efficiently limited by modifying the material surface properties through the control of physical-chemical parameters such as steric hindrance, hydrophobicity, van der Waals forces, or electrostatic interactions. ${ }^{40-44}$ Nature offers multiple examples of fouling control strategies employed by animal and plants, which involve different physical-chemical approaches. The latter are often associated with the presence of micro/ nanopatterned surface topographies characterized by various diameters, widths, lengths, heights, and pitches. ${ }^{40,45-47}$ Laboratory research has been oriented toward mimicking these morphologies, finding that tuning the micro/nanostructure parameters may prevent surface colonization by specific microorganisms. ${ }^{45,46}$ Many organic biomaterials have been tested, including, among others, poly(dimethylsiloxane), polyurethane, cellulose, poly(ethylene glycol), and thiophenebased conducting polymers. ${ }^{45,47,48}$ Surprisingly, the endless possibilities offered by silk manufacturing and processing techniques have just started to gain attention. In a very recent work by $\mathrm{Chu}$ and colleagues, the nanostructuring of silk substrates was first reported for orthopedic implants. ${ }^{49}$ Moreover, it has been widely reported that proper modulation of the surface topography may strongly affect proliferation, migration, and stem cell differentiation processes. ${ }^{50}$ This may open interesting perspectives toward the realization of a double-functional device, endowing antifouling properties and mechanical cues for specific cell-substrate interaction and metabolism modulation.

In this work, we report the preparation of micro- and nanopatterned silk surface topographies and the quantification of the adhesion grade of GFP-encoded Escherichia coli (E. coli) bacteria by means of fluorescence microscopy imaging. We observe a reduction in bacterial adhesion on structured surfaces up to $66 \%$ as compared with unpatterned substrates. Importantly, this is not accompanied by alteration in the cytocompatibility of the patterned silk substrate, as evaluated by viability assays with mammal cell model cultures. In view of possible applications in the biophotonics field, we also couple silk-based substrates to light-sensitive organic semiconducting polymer with distinct optoelectronics properties. We verify that the coating with the polymer does not hinder the antifouling effect shown by the patterned silk substrates. Our work offers interesting perspectives in the realization of novel silk-based biocompatible devices, endowed with both antifouling and photonics functionalities.

\section{EXPERIMENTAL SECTION}

2.1. Materials and Silk Substrates Fabrication. Regioregular poly(3-hexylthiophene-2,5-diyl) (rr-P3HT) (purity 99.995\%, molecular weight $15000-45000$ ), o-dichlorobenzene, Dulbecco's modified Eagle's medium (DMEM), Trypsin-EDTA, penicillin, phosphate buffer saline (PBS) tablets, streptomycin, fibronectin (from bovine plasma), 3-(4,5-dimethylthiazol-2-yl)-2,5-diphenyltetrazolium bromide (MTT), LiBr, Ampicillin, and $\mathrm{EtOH}$ were purchased from Sigma-Aldrich. PDMS elastomer (Sylgard 184) was purchased from Dow Corning. Fetal bovine serum (FBS) was purchased from Euroclone. All chemicals were used without any further purification.

For the preparation of silk fibroin films, Bombyx mori degummed silk fibers $(2 \mathrm{~g})$ were dissolved in a $\mathrm{LiBr}$ solution $(9.3 \mathrm{M}, 10 \mathrm{~mL})$ at $60{ }^{\circ} \mathrm{C}$ for $90 \mathrm{~min}$ obtaining a $20 \%(\mathrm{w} / \mathrm{v})$ solution. Dialysis tubing (molecular weight cutoff of $12000 \mathrm{Da}$ ) was used to dialyze the solution in water for $48 \mathrm{~h}$ at room temperature to remove the $\mathrm{LiBr}$ salt. The aggregates formed during dialysis were removed by centrifugation (4000 rpm, $25 \mathrm{~min}$ ). After these steps, the aqueous silk solution concentration was approximately $6 \%(\mathrm{w} / \mathrm{v})$, as determined by gravimetric analysis performed on the dried sample. Silk solution was then cast on both flat and patterned PDMS master molds and dried in a fume hood under gentle aspiration for $24 \mathrm{~h}$, following the procedure described in ref 51. The patterned PDMS films were obtained by replica molding of the polycarbonate layer of a CD-ROM (to obtain the master for the nano stripes) and of honeycomb structured layers prepared by the breath figure templating technique (to obtain the masters for the microwells). The polymeric materials used and conditions of the breath figure process were defined in order to obtain two different kinds of microporous templates, with larger $(\sim 5 \mu \mathrm{m})$ and smaller $(1-2 \mu \mathrm{m})$ microcavities, as described in ref 52 and ref 53 , respectively. Once solidified, the fibroin films were subjected to a water vapor annealing by placing them in a desiccator together with a water reservoir for $12 \mathrm{~h}$. This process increases the crystallinity of the silk-based material and makes it insoluble in water. ${ }^{54}$ Then, the dry fibroin films were softened by 2 min immersion in distilled water and carefully detached from the PDMS master.

For the preparation of polymer-coated silk substrates, fibroin silk films were anchored on a glass slide using adhesive tape and air-dried for one night. rr-P3HT was dissolved in $o$-dichlorobenzene $\left(5 \mathrm{~g} \mathrm{~L}^{-1}\right)$ and stirred for one night at $50{ }^{\circ} \mathrm{C}$. rr-P3HT solution was then deposited on dry silk substrates by spin coating (speed $1500 \mathrm{rpm}$, acceleration $1600 \mathrm{rpm} \mathrm{s}^{-1}$ ) and the obtained samples were left in a vacuum for $40 \mathrm{~min}$ before use.

2.2. Substrate Characterization. Scanning electron microscopy (SEM) micrographs were acquired using a TESCAN MIRA III scanning electron microscope (operating voltage $4 \mathrm{kV}$, working distance $19 \mathrm{~mm}$, stage tilt angle $30^{\circ}$ ). Prior to SEM image acquisition, silk substrates were attached to glass slides using adhesive tape, airdried, and covered by a thin gold layer (thickness $6 \mathrm{~nm}, 1.5 \mathrm{Cr}$ adhesion layer) using a metal evaporator. The atomic force microscopy (AFM) images were acquired using an NT-MDT NTEGRA apparatus in tapping mode under ambient conditions. For water contact angle measurements, silk-based samples were attached to glass slides using adhesive tape and air-dried overnight. Then, photographs of $5 \mu \mathrm{L}$ water droplets deposited on the different substrates were taken using an optical contact angle measuring and contour analysis system (DataPhysics OCA 15EC). The calculation of the static water contact angle was carried out using the SCA 20 software (DataPhysics), according to the sessile drop method.

2.3. Cell Culture Preparation and Viability Assay. Silk substrates were sterilized by immersion in $\mathrm{EtOH} 70 \%$ for $3 \mathrm{~h}$ and washed 3 times with $\mathrm{mQ}-\mathrm{H}_{2} \mathrm{O}$. Then, a layer of fibronectin $(2 \mu \mathrm{g}$ $\mathrm{mL}^{-1}$ in PBS buffer solution) was deposited on the sample surface and incubated for $1 \mathrm{~h}$ at $37^{\circ} \mathrm{C}$, in order to promote cellular adhesion. Excess fibronectin was then removed by rinsing with PBS prior to cell plating. HEK-293 cells were cultured in cell culture flasks containing Dulbecco's modified Eagle's medium (DMEM) with $10 \%$ fetal bovine serum (FBS), $100 \mu \mathrm{g} \mathrm{L}^{-1}$ penicillin, and $100 \mu \mathrm{g} \mathrm{mL}^{-1}$ streptomycin. Culture flasks were maintained in a humidified incubator (Forma series II water jacketed $\mathrm{CO}_{2}$ incubator, Thermofisher) at $37^{\circ} \mathrm{C}$ with $5 \% \mathrm{CO}_{2}$. When at confluence, HEK-293 cells were enzymatically dispersed using trypsin-EDTA and then plated on the different samples at a concentration of 20000 cells cm$~^{-2}$ and maintained in the incubator at $37{ }^{\circ} \mathrm{C}$ with $5 \% \mathrm{CO}_{2}$. Cell proliferation was assessed after 24,72 , and $96 \mathrm{~h}$ in vitro by performing the MTT assay. For each time point, the growing medium was replaced with RPMI medium without phenol red containing $0.5 \mathrm{mg} \mathrm{mL}^{-1}$ of MTT, and the samples were maintained in the dark at $37{ }^{\circ} \mathrm{C}$ for $3 \mathrm{~h}$. Then, the medium was removed and the samples were air-dried at room temperature. Dry samples were subsequently immersed in $200 \mu \mathrm{L}$ of ethanol in order to dissolve the formazan salt produced by cells through reduction of MTT. The proliferation cell rate was calculated as the difference in 
absorbance at 560 and $690 \mathrm{~nm}$. Statistical significance was determined by the one-way ANOVA Dunnett's post hoc test.

2.4. Bacterial Cultures and Adhesion Evaluation. The gene encoding eGFP was PCR amplified from a pDONR-P2R-P3 vector carrying the eGFP coding sequence, and cloned into a pET23a $(+)$ by conventional methods using NdeI and XhoI restriction sites (Table 1). The resulting construct (pET23a(+)-eGFP) was sequence verified and transformed into E. coli Rosetta (DE3) competent cells (Invitrogen).

Table 1. Primer Sequence $\left(5^{\prime}-3^{\prime}\right)$ Used in This Study ${ }^{a}$ eGFP

$\begin{array}{ll}\text { Forward }_{\text {Nat }} & \text { ccccccatatggtgagcaagggcgaggagc } \\ \text { Reverse }_{1} & \text { ggtctggctcgaggtacagctcgtccatgcc }\end{array}$

${ }^{a}$ The restriction sites for NdeI (forward) and Xhol (reverse) are underlined.

Bacterial cells were cultured as previously described. ${ }^{55}$ Briefly, a single colony of bacteria carrying the pET23a $(+)$-eGFP plasmid was inoculated in Luria-Bertani (LB) broth in the presence of ampicillin $(50 \mu \mathrm{g} / \mathrm{mL})$ and incubated overnight at $37^{\circ} \mathrm{C}$ until stationary phase was reached. The bacterial culture was then diluted to $\mathrm{OD}_{600}=0.1$ (1 $\times 10^{7} \mathrm{CFU} \mathrm{mL}^{-1}$ ) in LB media supplemented with the same antibiotics. Then, each substrate was incubated with $5 \mathrm{~mL}$ of bacterial suspension and kept in 12-well culture plates. Finally, isopropyl-1thio-D-galactopyranoside (IPTG) was added to the bacterial culture at a final concentration of $1 \mathrm{mM}$, following a $24 \mathrm{~h}$ incubation at $25{ }^{\circ} \mathrm{C}$ on a platform shaker. After $24 \mathrm{~h}$, the samples were removed from the bacteria growing medium, washed 3 times with $\mathrm{mQ}-\mathrm{H}_{2} \mathrm{O}$, and covered with a drop of water. Fluorescence images for the evaluation of the bacteria adhesion grade on the different substrates were acquired using an inverted fluorescence microscope (Nikon Eclipse $\mathrm{Ti}$ ), by exciting with a $470 \mathrm{~nm}$ light source (Lumencor Spectra $\mathrm{X}$ ) and using a standard FITC filters set. The average number of bacteria adhered on each substrate type was quantified using ImageJ software. Mean values were averaged over $n=10$ fields of view for each sample type, and over $n=3$ statistically independent samples. For evaluating the bactericidal activity, bacterial cells were cultured as described above. However, no IPTG was added into the bacterial solution. The optical density of these solutions was measured at $600 \mathrm{~nm}$ using a
UV-vis spectrometer. Control measurements on bacteria grown in the medium where no sample is present were carried out at the same time.

\section{RESULTS AND DISCUSSION}

3.1. Fabrication of Micropatterned Silk Substrates. We fabricate different micro- and nanopatterned silk fibroin substrates by employing a soft-lithography approach. ${ }^{51}$ The procedure, sketched in Figure 1, starts with the extraction of the silk fibroin protein from the Bombyx mori degummed silk fibers through dissolution in $\mathrm{LiBr}$ solution and subsequent dialysis against ultrapure water. The silk fibroin solution is then drop-casted on top of micro- and nanopatterned PDMS molds. Once solidified, the fibroin films are subjected to water vapor annealing. This process increases the crystallinity by promoting the formation of $\beta$-sheet dominated silk II structure, which is water-insoluble. $^{54}$ Four types of surface morphology are obtained by replicating different PDMS molds: flat nanostripes, small $\mu$ wells, and big $\mu$ wells. To obtain the PDMS mold for nanostriped silk, we replicated the grooved polycarbonate layer of a CD-ROM, while for fabricating the microdomes mold (negative morphology for $\mu$ wells), we employed the natureinspired templating process known as breath figure formation. ${ }^{56}$ Therefore, not only the preparation of silk substrates but the whole patterning process are achieved by affordable and simple techniques based on self-assembly. For the flat controls, a flat PDMS layer is used. Silk fibroin solution is initially processed into $\sim 20 \mathrm{~cm}^{2}$ films (Figure 1 top inset), which are then cut into $1.8-\mathrm{cm}$-diameter disks for performing the experiments (Figure 1 bottom inset). The thickness of the micro- and nanopatterned silk films is about $100 \mu \mathrm{m}$. Such a thickness is significantly higher than the height of the nanoand microfeatures present on the patterned silk surfaces. This fact suggests that the mechanical properties of the patterned substrates are comparable to those reported in the literature for flat films obtained with the same fabrication process. ${ }^{54}$ Moreover, the micro- and nanopatterned coatings do not

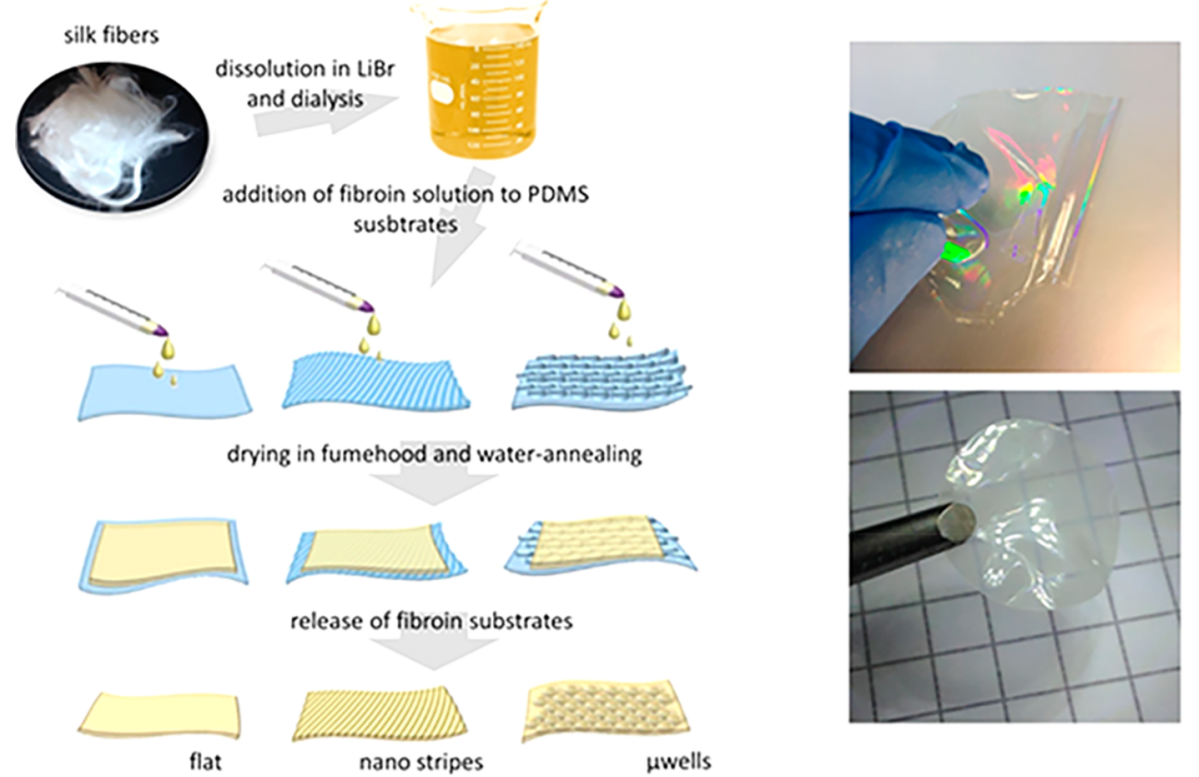

Figure 1. Silk substrate fabrication. A soft lithography and self-assembly approach allows for fast, scalable, and highly repeatable fabrication of patterned silk substrates, with different designs. In this case, nanostripes and microwells with different diameters have been realized. In the inset: representative photographs of a patterned silk film before cutting (top) and a 1.8-cm-diameter disk used for the assays (bottom). 
negatively affect the silk mechanical properties, since all reported patterned substrates can be easily bent at curvature angles $\gg 90^{\circ}$, even when they are in the dry form, and the wet coatings can be successfully wrapped on different curved objects (Figure S1).

Figure 2 shows the surface topography of the planar, unpatterned silk substrates (Figure 2a) and of micro/

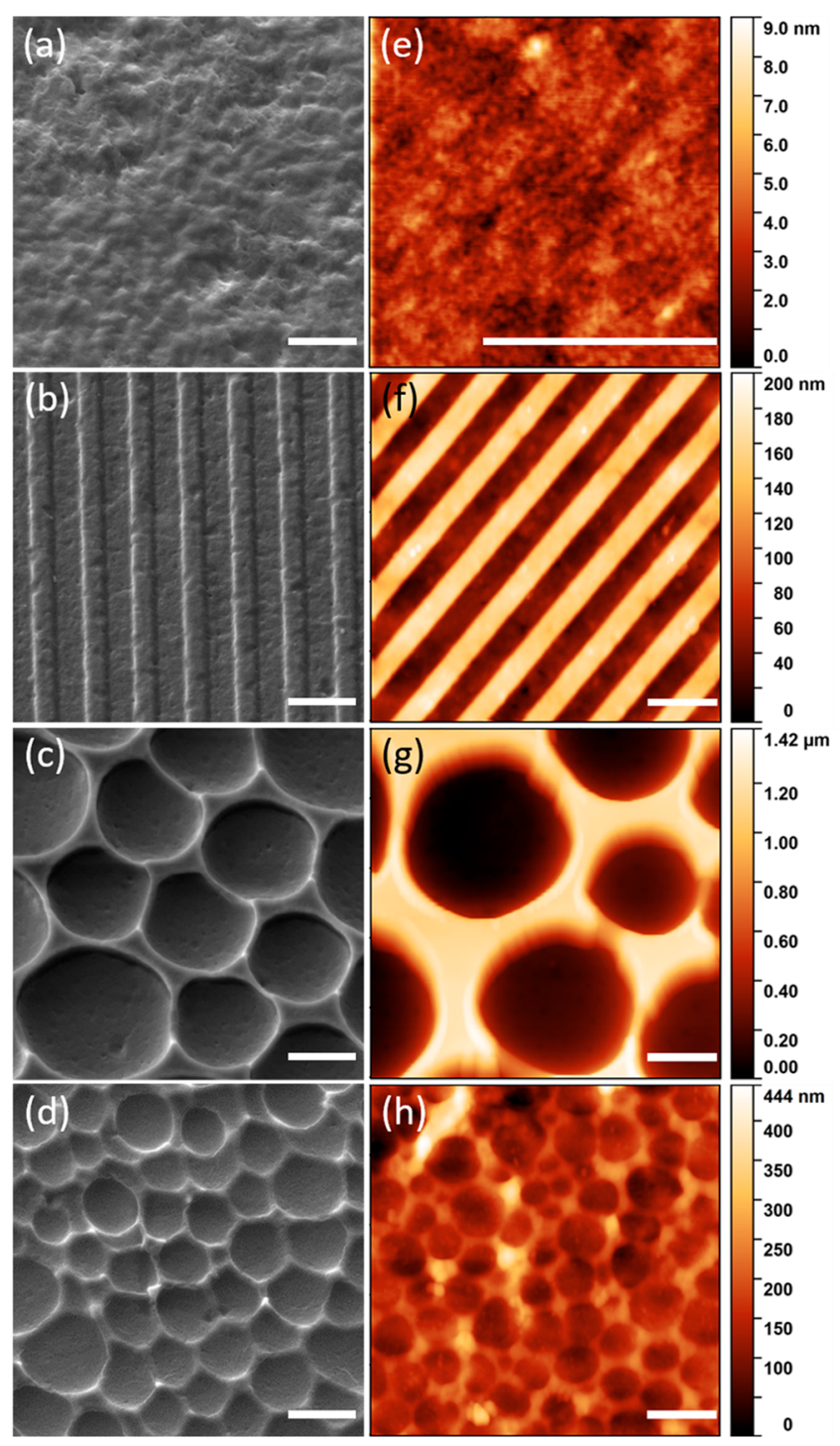

Figure 2. Topography of flat and micro/nanopatterned silk substrates. (a) -(d): SEM top-view images of silk flat (a), nanostripes (b), $\mu$ wells 1 (c), and $\mu$ wells 2 (d). Scale bars, $2 \mu \mathrm{m}$. (e)-(h): AFM topography images of silk flat (e), nanostripes (f), $\mu$ wells 1 (g), and $\mu$ wells 2 (h) samples. Scale bars, $2 \mu \mathrm{m}$.

nanostructured samples with three different geometries (Figure $2 \mathrm{~b}-\mathrm{d}$ ), as obtained by acquiring SEM micrographs in top-view configuration. By employing different PDMS molds, three topographies are obtained: (1) a nanostripe-patterned silk fibroin surface, composed of grooves of $\sim 800 \mathrm{~nm}$ width and spacing of the same dimension (nanostripes, Figure 2b); (2) silk fibroin microwells of different diameters, ranging from 3.5 to $5 \mu \mathrm{m}$ ( $\mu$ wells 1 , Figure $2 \mathrm{c}$ ); (3) silk fibroin microwells with a shape similar to $\mu$ wells 1 , but lower diameter, in the range $1-$ $2 \mu \mathrm{m}$ ( $\mu$ wells 2 , Figure $2 \mathrm{~d}$ ). The choice of these patterning geometries was inspired by antifouling approaches adopted by nature systems, ${ }^{47}$ as well as by existing literature reports about biomimetic antifouling devices. ${ }^{39,42}$ In particular, nanostripes and $\mu$ wells 2 follow the solution adopted, for instance, in the shark skin, in which grooves of width lower than the bacterial dimensions lead to inhibition of biofilm formation. The geometry $\mu$ wells 1 instead was optimized in order to induce the formation of air pockets within the microstructures, which was previously reported to substantially inhibit bacterial adhesion $^{57}$ and recalls solutions adopted, for instance, in the lotus leaf. ${ }^{47}$ Silk substrate morphology is also characterized by AFM (Figure $2 \mathrm{e}-\mathrm{h}$ ). The surface roughness of the flat silk control substrates (Figure 2e) is in line with the existing literature (root-mean-square roughness (RMS) value \pm standard error of the mean (s.e.m.), $0.86 \pm 0.03 \mathrm{~nm}) .{ }^{58}$ The average height of the microstructured features amounts at $0.124 \pm 0.005 \mu \mathrm{m}, 1.06 \pm 0.03 \mu \mathrm{m}$, and $0.122 \pm 0.007 \mu \mathrm{m}$ (mean \pm s.e.m.) for the nanostripe, $\mu$ well 1 , and $\mu$ well 2 cases, respectively (Figure $2 \mathrm{f}-\mathrm{h}$ ).

3.2. HEK-293 Cells Cultures on Micro/Nanopatterned Silk Substrates. Once extracted from Bombyx mori silk following an established purification route, ${ }^{59}$ silk fibroin shows optimal biocompatibility, as documented in several in vivo and in vitro studies. ${ }^{60-62}$ However, much less is known about the cytocompatibility of patterned silk fibroin substrates. Thus, by culturing HEK-293 cell models on top of the different microand nanopatterned silk fibroin-based substrates, we directly investigate whether structured surface topographies sizably affect the viability of living cells. Cell proliferation is monitored by performing the MTT assay (Figure 3). The method is based

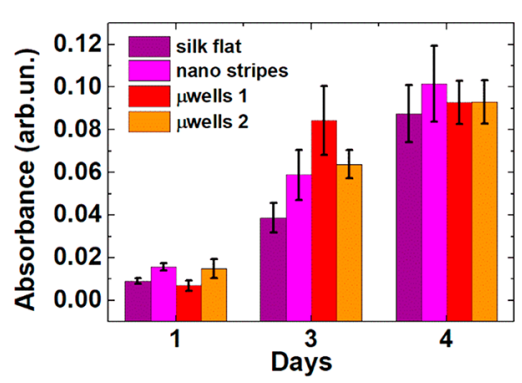

Figure 3. HEK-293 cells viability. MTT viability assay on HEK293 cells plated on top of the different silk substrates. Data are reported as mean \pm s.e.m. A one-way ANOVA followed by post hoc Dunnett's correction analysis reveals that none of the patterned silk substrates present any statistically significant difference in comparison to the planar silk samples.

on the use of the water-soluble tetrazolium salt (MTT), which is metabolized/reduced by living cells into a water-insoluble formazan product. As the optical absorption of formazan, which has a characteristic purple color, is proportional to the number of living cells, it can be directly related to the cell viability and capability to proliferate. Formazan absorption in the MTT-treated cell cultures on the different morphologies is evaluated after 1,3 , and 4 days in vitro (DIV), showing no statistically significant differences between micro/nanopatterned and planar silk topographies cases (Figure 3). This clearly indicates that the micro- and nanostructures do not substantially affect the viability and the proliferation of HEK293 cells, despite the fact that cells may be subjected to a higher surface pressure due to the reduced contact area. Instead, conditions favorable to cell proliferation are established, as also reported by recent literature and attributed 

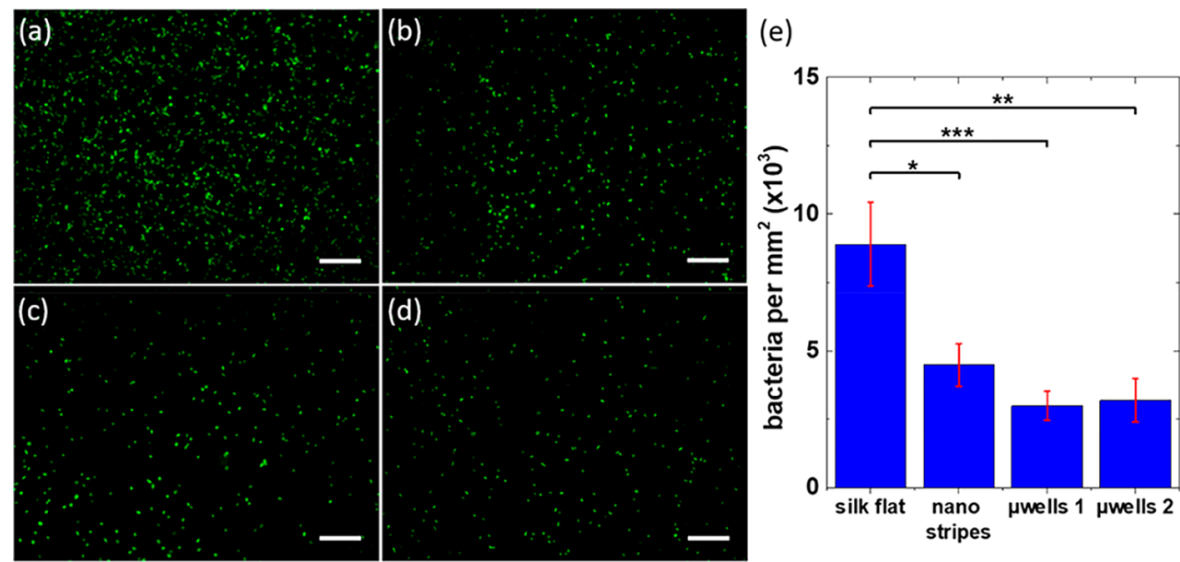

Figure 4. Evaluation of bacterial adhesion on patterned silk. Fluorescent microscopy images of GFP expressing bacteria cultured on flat (a), nanostriped (b), $\mu$ wells 1-patterned (c), and $\mu$ wells 2-patterned (d) silk substrates, after $24 \mathrm{~h}$. Scale bars, $50 \mu \mathrm{m}$. (e) Quantitative analysis of bacteria observed in a-d. $* p<0.05, * * p<0.01, * * * p<0.001$.

to the higher surface energy and subsequent superhydrophilicity typical of the silk substrate. ${ }^{49}$ In our case, there are no significant differences between flat and patterned silk substrates, but this observation may critically depend on the considered cell model, and it should not be considered of general validity. Interestingly, an enhancement in the proliferation of cells that were seeded on nanopatterned silk was recently observed in the case of human adipose mesenchymal stem cells, which usually feature exquisite sensitivity to the underlying substrate topography. ${ }^{63}$

3.3. Evaluation of Escherichia coli Adhesion on Pristine and Structured Silk Substrates. The antifouling capability of the different micro/nanopatterned silk samples is evaluated by employing the E. coli bacterial model, genetically modified to express green fluorescent protein (GFP), which is used as a visual marker. The substrates are incubated with the GFP-expressing E. coli for $24 \mathrm{~h}$, washed with ultrapure water for removing all unattached bacteria and covered with an ultrapure water drop. The number of adherent bacteria is then quantified by acquiring GFP fluorescence emission (Figure $4 \mathrm{a}-\mathrm{d}$ ) using a fluorescence microscope. Measurements were acquired after $24 \mathrm{~h}$, when biofilm formation is known to be irreversible and fully completed. ${ }^{64}$ All the silk samples display a lower number of adhered bacteria in comparison to the flat silk control. Importantly, the micro- and nanopatterned silk samples present a significant reduction in the number of $E$. coli attached to their surfaces in comparison with the flat silk morphology, amounting to $49 \%(p<0.05), 66 \%(p<0.001)$, and $64 \%(p<0.01)$ for the nanostripes, $\mu$ wells 1 , and $\mu$ wells 2 , respectively (Figure 4e). For completeness, we verified by AFM that the patterning structures are preserved after exposure to the media employed for preparing the cell and bacterial cultures (Figure S2).

This result can be attributed to a topography-driven antifouling mechanism. In previous reports, two main effects were found to have a central role in the inhibition of bacterial adhesion: (1) a steric effect due to the presence of a regular micropatterned morphology characterized by a features size lower than bacterial dimension ${ }^{39,40,65}$ and (2) the hydrophobic effect induced by microstructured surfaces. ${ }^{39,41,57,66}$ In both cases, the driving force of the antifouling process relies on the reduction of the surface area to which the organisms can adhere. The reduced bacterial adhesion achieved with silk nanostripes and $\mu$ wells 2 can be explained by considering the former mechanism, since the E. coli average length $(\sim 2 \mu \mathrm{m})$ and diameter $(\sim 1 \mu \mathrm{m})^{67,68}$ are higher than the width of the nanostripe silk grooves $(\sim 800 \mathrm{~nm})$, as well as the $\mu$ wells 2 mean diameter (between 1 and $2 \mu \mathrm{m}$ ). On the contrary, this argumentation is not valid for explaining the bacterial adhesion reduction induced by $\mu$ wells 1 , because the diameter of the wells that compose this architecture (in the range between 3.5 and $5 \mu \mathrm{m}$ ) is about twice as large as the E. coli average dimension. In order to clarify the origin of the antifouling effect observed, experiments for the evaluation of the hydrophobicity of the samples are carried out by measuring the static contact angle of the considered surfaces (Figure 5

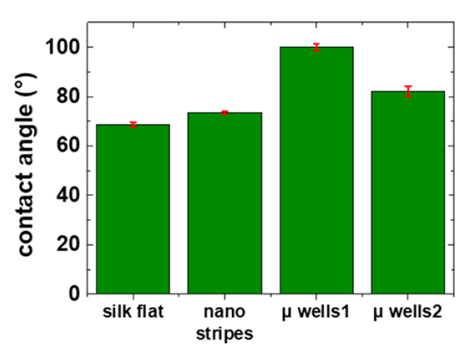

Figure 5. Water contact angle measurement. Static contact angles established by water drops over planar and micro/nanopatterned silk morphologies.

and Figure S3). Generally, the limit between hydrophilicity and hydrophobicity is placed at a contact angle value of $90^{\circ} .{ }^{69}$ Planar silk is hydrophilic, presenting a water contact angle value of $69^{\circ} \pm 1^{\circ}$. Silk nanostripe and $\mu$ well 2 samples show increased water contact angle values $\left(73^{\circ} \pm 0.5^{\circ}\right.$ and $82^{\circ} \pm 2^{\circ}$, respectively), but they still fall in the hydrophilic regime, confirming that the observed decrease of E. coli adhesion is due to a steric effect exerted by the surface topography, and it is not strictly related to the surface wettability. $\mu$ Well 1 substrates display instead a hydrophobic behavior, with a water contact angle of $100^{\circ} \pm 1^{\circ}$. This result is in line with the existing literature, where similar honeycomb surface topographies have been shown to influence the wettability of polymer films. ${ }^{57,70,71}$ In particular, this behavior has been explained by the formation of air pockets between the surface of the substrate and the water droplets, when the latter are much larger than the dimension of the structures present on the surface of the 
substrate. $^{70}$ This phenomenon could explain the $\mu$ well 1 antifouling properties, since it directly determines the amount of surface that is available for bacterial attachment. Our interpretation of the results for $\mu$ wells 1 is also in line with the work by Manabe et al., showing that when the size of the pores lies between 3.5 and $11 \mu \mathrm{m}$ and the contact angle of the culture medium is high, bacteria experience a limited contact with the surface. ${ }^{57}$

3.4. Evaluation of $E$. coli Adhesion on rr-P3HTCovered Silk Substrates. Among the numerous biotechnology applications of silk-based biomaterials, optics and biophotonics are emerging as some of the most attractive ones. Various types of implantable optical devices, including optical waveguides, ${ }^{14,72,73}$ optical fibers, ${ }^{74}$ and microprism arrays, ${ }^{75,76}$ based on biocompatible silk have been described in the literature. ${ }^{13,77}$ This is due to silk's good optical transparency, which ensures a high refractive index and low optical losses, even when used within in vivo systems. Silk has also been recently used as a long-term biocompatible substrate in the fabrication of functionally autonomous photovoltaic visual prostheses. ${ }^{62}$ In this case, the excellent mechanical properties of silk were usefully coupled to the light responsivity and charge generation properties of a thiophene-based conjugated polymer, regioregular poly(3-hexylthiophene-2,5-diyl) (rr$\mathrm{P} 3 \mathrm{HT}$ ). It has been shown that a silk/polymer device is highly tolerated within the subretinal space of rats and partially recovers visual acuity in blind retinas. ${ }^{62,78,79}$ These seminal works demonstrated that silk-based biomaterials can be coupled to optically active polymers, thus providing a vast array of opportunities for the development of light-based diagnostic and therapeutic tools. In view of its huge in vivo application potential, we set out to investigate whether the excellent antifouling efficacy shown by microstructured silk substrates is preserved even in the presence of a thin film of a semiconducting polymer deposited on top of the surface. We focused our attention on the substrate that gave the best antifouling result, $\mu$ wells 1 , and on the planar silk morphology as a control. The deposition of the layer of the semiconducting polymer is carried out by spin-coating, by selecting the proper rr-P3HT concentration and spin parameters that do not lead to a passivation of the microstructured topography. Polymer thin film thickness is on the order of $30 \mathrm{~nm}$, showing an optical absorption of $\sim 0.3$, which ensures good charge generation efficiency and photovoltaic properties also when exposed to aqueous saline solutions and cell growth media. ${ }^{80}$ SEM images of the rr-P3HT-covered/uncovered samples confirm that the honeycomb microstructure is not altered upon polymer deposition (Figure S4). Moreover, photographs and fluorescence images corroborate this picture and highlight the uniformity of the P3HT coverage (Figure S5). E. coli adhesion is evaluated in rr-P3HT-covered silk samples (Figure 6), after having verified their stability in the culture media (Figure S6). We notice that the average density of bacteria adherent to polymer-coated silk flat samples is similar to the one recorded in uncoated samples (Figure 4), thus allowing us to exclude a concomitant antifouling action due to the polymer thin film alone. We also notice that the hydrophobicity value typical of rr-P3HT polymer thin films in dry conditions (about $100^{\circ}$ ) is substantially decreased when the polymer is directly exposed to an aqueous environment, due to water permeation and ion doping, dropping up to $60-70^{\circ} .{ }^{81}$ Thus, the polymer itself is not expected to sustain or to boost an antifouling effect. Importantly, however, the $\mu$ wells $1 / \mathrm{P} 3 \mathrm{HT}$ sample display a

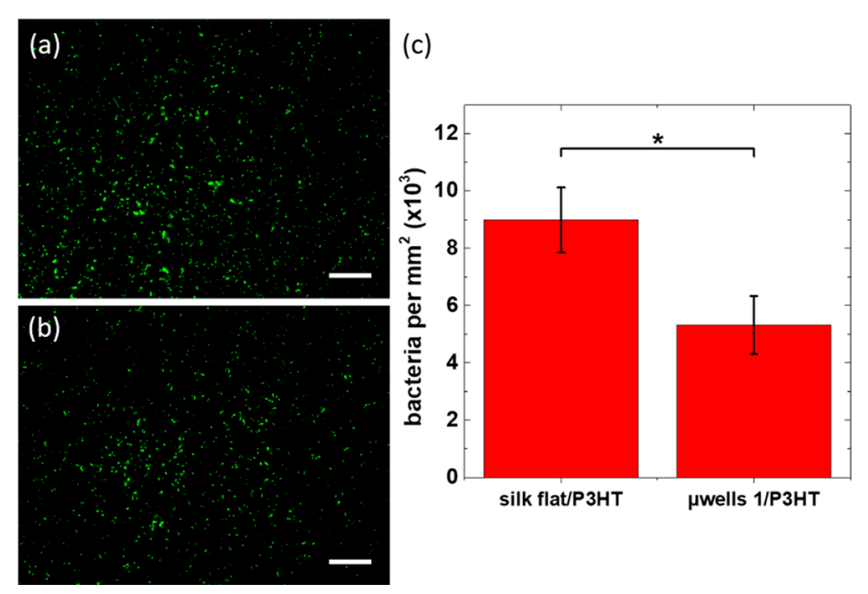

Figure 6. Evaluation of bacteria adhesion. Fluorescent microscopy images of GFP expressing bacteria cultured on silk flat/P3HT (a) and $\mu$ well 1/P3HT (b), after $24 \mathrm{~h}$. Scale bar $50 \mu \mathrm{m}$. (c) Quantitative analysis of bacteria observed in $(\mathrm{a}, \mathrm{b}) .{ }^{*} p<0.05$.

reduction of $41 \%(p<0.05)$ in the adhesion of bacteria relative to the flat control, thus demonstrating that the topographydriven antifouling effect of structured silk is substantially preserved also in the presence of a thin film of a conjugated polymer deposited on top of its upper, bacteria-exposed surface.

In order to corroborate our findings, we carried out experiments aimed at the quantification of the bacterial growth inside the culture medium in the presence of all the tested substrates, thus providing an insight into the existence of possible sample-induced bactericidal effects. E. coli cultures are prepared in the same way as in the antifouling experiments case, and the bacterial growth is evaluated by measuring the culture medium optical absorption at $600 \mathrm{~nm}$ after $24 \mathrm{~h}$ (Figure 7). The results show that there are no significant differences between the case where no substrate is present and the one where bacteria are incubated with both P3HTuncovered (Figure 7a) and P3HT-covered (Figure 7b) silk samples. On the basis of these findings, we can conclude that the reduction of the surface-attached bacteria is only due to an unfavorable bacterial adhesion induced by the micro/nanopatterned topographies and we can exclude a bactericidal effect exerted by the micro/nanopatterned silk-based morphologies.

\section{CONCLUSIONS}

A fast, facile, and easily scalable processing method based on soft lithography is applied to realize nano- and microstructured silk samples, with different geometries. We show that patterned silk substrates display excellent antifouling properties, leading in all cases to a substantial reduction of bacterial adhesion. At the same time, we show that the well-known cytocompatibility properties, which have been widely assessed in multiple studies for silk flat substrates, are fully preserved also in the case of silk microstructures. The antifouling effect is explained on the basis of a complex interplay between geometrical constraints, as the bacteria membrane has a lower surface area available for adhesion, and the enhancement of hydrophobicity induced by microstructuring. In particular, we identify an optimal combination of the above-mentioned effects, and we demonstrate that suitable silk patterning leads to a more than $65 \%$ decrease in bacteria adhesion, as compared to flat silk substrates. As an important step toward the realization of 
(a)

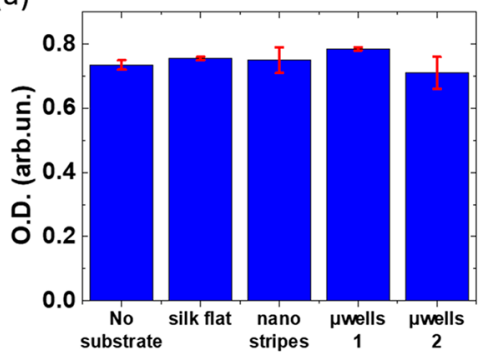

(b)

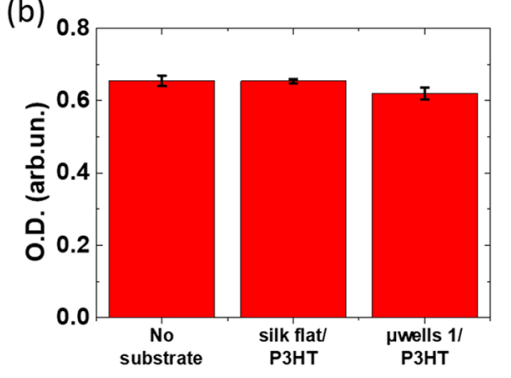

Figure 7. Evaluation of the antibacterial activity of the micro/ nanopatterned silk-based substrates. Bacterial growth in solution after $24 \mathrm{~h}$ incubation with P3HT-uncovered (a) and P3HT-covered (b) silk substrates in comparison to the case where no substrate is present inside the growth medium. Bacterial growth is evaluated as the optical density (O.D.) at $600 \mathrm{~nm}$.

fully biocompatible and functional devices for photomedicine, we demonstrate that the antifouling properties of patterned silk microstructures are fully preserved also in combination with a light-active conjugated polymer. This paves the way toward the realization of a novel generation of biopolymer implantable devices, endowed with mechanical properties similar to those of biological tissues, and able to both optically modulate/ optically sense the activity of cells and to substantially inhibit biofilm formation.

\section{ASSOCIATED CONTENT}

\section{SI Supporting Information}

The Supporting Information is available free of charge at https://pubs.acs.org/doi/10.1021/acsami.9b18187.

Representative photographs showing the micro/nano patterned silk substrates in different configurations and water droplets in contact with the silk-based substrates. AFM images showing the patterned silk topographies after incubation in culture media. SEM top-view images of the bare and P3HT-covered silk substrates. Representative photographs and fluorescence images of the P3HT layer deposited on patterned silk. Representative photographs and photoluminescence spectra of the P3HT-covered substrates after incubation in culture media. (PDF)

Stretchability of micropatterned silk fibroin substrates (AVI)

\section{AUTHOR INFORMATION}

\section{Corresponding Author}

M. R. Antognazza - Center for Nano Science and Technology@ PoliMi, Istituto Italiano di Tecnologia, Milano 20133, Italy; () orcid.org/0000-0003-4599-2384;

Email: mariarosa.antognazza@iit.it

\section{Other Authors}

G. Tullii - Center for Nano Science and Technology@PoliMi, Istituto Italiano di Tecnologia, Milano 20133, Italy; Department of Physics, Politecnico di Milano, Milano 20133, Italy; (i) orcid.org/0000-0002-6595-3449

S. Donini - Center for Nano Science and Technology@PoliMi, Istituto Italiano di Tecnologia, Milano 20133, Italy

C. Bossio - Center for Nano Science and Technology@PoliMi, Istituto Italiano di Tecnologia, Milano 20133, Italy

F. Lodola - Center for Nano Science and Technology@PoliMi, Istituto Italiano di Tecnologia, Milano 20133, Italy; (-) orcid.org/0000-0002-3506-5619

M. Pasini - Istituto di Scienze e Tecnologie Chimiche "Giulio Natta", Consiglio Nazionale delle Ricerche (SCITEC-CNR), Milano 20133, Italy; 이이이.org/0000-0002-7811-6367

E. Parisini - Center for Nano Science and Technology@PoliMi, Istituto Italiano di Tecnologia, Milano 20133, Italy; (1) orcid.org/0000-0002-5529-0039

F. Galeotti - Istituto di Scienze e Tecnologie Chimiche "Giulio Natta", Consiglio Nazionale delle Ricerche (SCITEC-CNR), Milano 20133, Italy; () orcid.org/0000-0003-4793-9827

Complete contact information is available at:

https://pubs.acs.org/10.1021/acsami.9b18187

\section{Author Contributions}

G.T., M.P., E.P., F.G., and M.R.A. planned the research. F.G. prepared the silk substrates. G.T. carried out SEM, AFM with help from F.G., fluorescence microscopy, cells viability assay with help from F.L., and contact angle measurements. S.D. prepared and characterized bacteria cultures. C.B. and F.L. helped with cell culture preparation. G.T. carried out data analysis. G.T. and M.R.A. interpreted the data and wrote the main manuscript, with contribution from all authors. M.R.A. supervised the work. All authors approved the final manuscript.

\section{Funding}

M.R.A. acknowledges support by the European Research Council (ERC) under the European Union's Horizon 2020 research and innovation program "LINCE", grant agreement no 803621. F.L. and M.R.A. acknowledge support by EU Horizon 2020 FETOPEN-2018-2020 Programme "LIONHEARTED”, grant agreement no 828984 .

\section{Notes}

The authors declare no competing financial interest.

\section{REFERENCES}

(1) Lawrence, B. D.; Marchant, J. K.; Pindrus, M. A.; Omenetto, F. G.; Kaplan, D. L. Silk Film Biomaterials for Cornea Tissue Engineering. Biomaterials 2009, 30 (7), 1299-1308.

(2) Kim, H. J.; Kim, U.-J.; Kim, H. S.; Li, C.; Wada, M.; Leisk, G. G.; Kaplan, D. L. Bone Tissue Engineering with Premineralized Silk Scaffolds. Bone 2008, 42 (6), 1226-1234.

(3) Fuchs, S.; Jiang, X.; Schmidt, H.; Dohle, E.; Ghanaati, S.; Orth, C.; Hofmann, A.; Motta, A.; Migliaresi, C.; Kirkpatrick, C. J. Dynamic Processes Involved in the Pre-Vascularization of Silk Fibroin Constructs for Bone Regeneration Using Outgrowth Endothelial Cells. Biomaterials 2009, 30 (7), 1329-1338.

(4) Unger, R. Growth of Human Cells on a Non-Woven Silk Fibroin Net: A Potential for Use in Tissue Engineering. Biomaterials 2004, 25 (6), 1069-1075.

(5) Wang, Y.; Bella, E.; Lee, C. S. D.; Migliaresi, C.; Pelcastre, L.; Schwartz, Z.; Boyan, B. D.; Motta, A. The Synergistic Effects of 3-D Porous Silk Fibroin Matrix Scaffold Properties and Hydrodynamic Environment in Cartilage Tissue Regeneration. Biomaterials 2010, 31 (17), 4672-4681. 
(6) Guarino, V.; Benfenati, V.; Cruz-Maya, I.; Saracino, E.; Zamboni, R.; Ambrosio, L. Instructive Proteins for Tissue Regeneration. In Functional 3D Tissue Engineering Scaffolds; Elsevier, 2018; pp 23-49. DOI: 10.1016/B978-0-08-100979-6.00002-1.

(7) Pistone, A.; Sagnella, A.; Chieco, C.; Bertazza, G.; Varchi, G.; Formaggio, F.; Posati, T.; Saracino, E.; Caprini, M.; Bonetti, S.; Toffanin, S.; Di Virgilio, N.; Muccini, M.; Rossi, F.; Ruani, G.; Zamboni, R.; Benfenati, V. Silk Fibroin Film from Golden-Yellow Bombyx Mori Is a Biocomposite That Contains Lutein and Promotes Axonal Growth of Primary Neurons: Yellow SF in the Field of Neuroregenerative Medicine. Biopolymers 2016, 105 (5), 287-299.

(8) Koh, L.-D.; Yeo, J.; Lee, Y. Y.; Ong, Q.; Han, M.; Tee, B. C.-K. Advancing the Frontiers of Silk Fibroin Protein-Based Materials for Futuristic Electronics and Clinical Wound-Healing (Invited Review). Mater. Sci. Eng., C 2018, 86, 151-172.

(9) Suzuki, S.; Shadforth, A. M. A.; McLenachan, S.; Zhang, D.; Chen, S.-C.; Walshe, J.; Lidgerwood, G. E.; Pébay, A.; Chirila, T. V.; Chen, F. K.; Harkin, D. G. Optimization of Silk Fibroin Membranes for Retinal Implantation. Mater. Sci. Eng., C 2019, 105, 110131.

(10) Germershaus, O.; Werner, V.; Kutscher, M.; Meinel, L. Deciphering the Mechanism of Protein Interaction with Silk Fibroin for Drug Delivery Systems. Biomaterials 2014, 35 (10), 3427-3434.

(11) Meinel, L.; Kaplan, D. L. Silk Constructs for Delivery of Musculoskeletal Therapeutics. Adv. Drug Delivery Rev. 2012, 64 (12), $1111-1122$.

(12) Li, A. B.; Kluge, J. A.; Guziewicz, N. A.; Omenetto, F. G.; Kaplan, D. L. Silk-Based Stabilization of Biomacromolecules. J. Controlled Release 2015, 219, 416-430.

(13) Humar, M.; Kwok, S. J. J.; Choi, M.; Yetisen, A. K.; Cho, S.; Yun, S.-H. Toward Biomaterial-Based Implantable Photonic Devices. Nanophotonics 2017, 6 (2), 1 DOI: 10.1515/nanoph-2016-0003.

(14) Parker, S. T.; Domachuk, P.; Amsden, J.; Bressner, J.; Lewis, J. A.; Kaplan, D. L.; Omenetto, F. G. Biocompatible Silk Printed Optical Waveguides. Adv. Mater. 2009, 21 (23), 2411-2415.

(15) Tsioris, K.; Tilburey, G. E.; Murphy, A. R.; Domachuk, P.; Kaplan, D. L.; Omenetto, F. G. Functionalized-Silk-Based Active Optofluidic Devices. Adv. Funct. Mater. 2010, 20 (7), 1083-1089.

(16) Benfenati, V.; Zamboni, R. Silk Biomaterials Enable Living Technologies Targeting Brain Cells. Nonlinear Opt. Quantum Opt. Concepts Mod. Opt. 2019, 50, 183-201.

(17) Sagnella, A.; Zambianchi, M.; Durso, M.; Posati, T.; Del Rio, A.; Donnadio, A.; Mazzanti, A.; Pistone, A.; Ruani, G.; Zamboni, R.; Benfenati, V.; Melucci, M. APTES Mediated Modular Modification of Regenerated Silk Fibroin in a Water Solution. RSC Adv. 2015, 5 (78), 63401-63406.

(18) Tao, H.; Kainerstorfer, J. M.; Siebert, S. M.; Pritchard, E. M.; Sassaroli, A.; Panilaitis, B. J. B.; Brenckle, M. A.; Amsden, J. J.; Levitt, J.; Fantini, S.; Kaplan, D. L.; Omenetto, F. G. Implantable, Multifunctional, Bioresorbable Optics. Proc. Natl. Acad. Sci. U. S. A. 2012, 109 (48), 19584-19589.

(19) Shimanovich, U.; Pinotsi, D.; Shimanovich, K.; Yu, N.; Bolisetty, S.; Adamcik, J.; Mezzenga, R.; Charmet, J.; Vollrath, F.; Gazit, E.; Dobson, C. M.; Kaminski Schierle, G.; Holland, C.; Kaminski, C. F.; Knowles, T. P. J. Biophotonics of Native Silk Fibrils. Macromol. Biosci. 2018, 18 (4), 1700295.

(20) Shan, D.; Gerhard, E.; Zhang, C.; Tierney, J. W.; Xie, D.; Liu, Z.; Yang, J. Polymeric Biomaterials for Biophotonic Applications. Bioact. Mater. 2018, 3 (4), 434-445.

(21) Zhang, Y.; Zhou, Z.; Fan, Z.; Zhang, S.; Zheng, F.; Liu, K.; Zhang, Y.; Shi, Z.; Chen, L.; Li, X.; Mao, Y.; Wang, F.; Sun, Y.-L.; Tao, T. H. Self-Powered Multifunctional Transient Bioelectronics. Small 2018, 14 (35), 1802050.

(22) Chatterjee, S.; Saxena, M.; Padmanabhan, D.; Jayachandra, M.; Pandya, H. J. Futuristic Medical Implants Using Bioresorbable Materials and Devices. Biosens. Bioelectron. 2019, 142, 111489.

(23) Hou, C.; Xu, Z.; Qiu, W.; Wu, R.; Wang, Y.; Xu, Q.; Liu, X. Y.; Guo, W. A Biodegradable and Stretchable Protein-Based Sensor as Artificial Electronic Skin for Human Motion Detection. Small 2019, 15 (11), 1805084.
(24) Tao, H.; Hwang, S.-W.; Marelli, B.; An, B.; Moreau, J. E.; Yang, M.; Brenckle, M. A.; Kim, S.; Kaplan, D. L.; Rogers, J. A.; Omenetto, F. G. Silk-Based Resorbable Electronic Devices for Remotely Controlled Therapy and in Vivo Infection Abatement. Proc. Natl. Acad. Sci. U. S. A. 2014, 111 (49), 17385-17389.

(25) Zhou, Z.; Shi, Z.; Cai, X.; Zhang, S.; Corder, S. G.; Li, X.; Zhang, Y.; Zhang, G.; Chen, L.; Liu, M.; Kaplan, D. L.; Omenetto, F. G.; Mao, Y.; Tao, Z.; Tao, T. H. The Use of Functionalized Silk Fibroin Films as a Platform for Optical Diffraction-Based Sensing Applications. Adv. Mater. 2017, 29 (15), 1605471.

(26) Seo, J.-W.; Kim, H.; Kim, K.; Choi, S. Q.; Lee, H. J. CalciumModified Silk as a Biocompatible and Strong Adhesive for Epidermal Electronics. Adv. Funct. Mater. 2018, 28 (36), 1800802.

(27) Huang, X. Bioresorbable Materials and Their Application in Electronics; Cambridge University Press, 2017.

(28) Kim, D.-H.; Viventi, J.; Amsden, J. J.; Xiao, J.; Vigeland, L.; Kim, Y.-S.; Blanco, J. A.; Panilaitis, B.; Frechette, E. S.; Contreras, D.; Kaplan, D. L.; Omenetto, F. G.; Huang, Y.; Hwang, K.-C.; Zakin, M. R.; Litt, B.; Rogers, J. A. Dissolvable Films of Silk Fibroin for Ultrathin Conformal Bio-Integrated Electronics. Nat. Mater. 2010, 9 (6), 511517.

(29) Tao, H.; Brenckle, M. A.; Yang, M.; Zhang, J.; Liu, M.; Siebert, S. M.; Averitt, R. D.; Mannoor, M. S.; McAlpine, M. C.; Rogers, J. A.; Kaplan, D. L.; Omenetto, F. G. Silk-Based Conformal, Adhesive, Edible Food Sensors. Adv. Mater. 2012, 24 (8), 1067-1072.

(30) Cha, G. D.; Kang, D.; Lee, J.; Kim, D. Bioresorbable Electronic Implants: History, Materials, Fabrication, Devices, and Clinical Applications. Adv. Healthcare Mater. 2019, 8 (11), 1801660.

(31) Zhou, Z.; Zhang, S.; Cao, Y.; Marelli, B.; Xia, X.; Tao, T. H. Engineering the Future of Silk Materials through Advanced Manufacturing. Adv. Mater. 2018, 30 (33), 1706983.

(32) Gomes, S. C.; Leonor, I. B.; Mano, J. F.; Reis, R. L.; Kaplan, D. L. Antimicrobial Functionalized Genetically Engineered Spider Silk. Biomaterials 2011, 32 (18), 4255-4266.

(33) Nilebäck, L.; Hedin, J.; Widhe, M.; Floderus, L. S.; Krona, A.; Bysell, H.; Hedhammar, M. Self-Assembly of Recombinant Silk as a Strategy for Chemical-Free Formation of Bioactive Coatings: A RealTime Study. Biomacromolecules 2017, 18 (3), 846-854.

(34) Lu, Z.; Xiao, J.; Wang, Y.; Meng, M. In Situ Synthesis of Silver Nanoparticles Uniformly Distributed on Polydopamine-Coated Silk Fibers for Antibacterial Application. J. Colloid Interface Sci. 2015, 452, $8-14$.

(35) Zhou, W.; Jia, Z.; Xiong, P.; Yan, J.; Li, Y.; Li, M.; Cheng, Y.; Zheng, Y. Bioinspired and Biomimetic AgNPs/Gentamicin-Embedded Silk Fibroin Coatings for Robust Antibacterial and Osteogenetic Applications. ACS Appl. Mater. Interfaces 2017, 9 (31), 25830-25846.

(36) Grainger, D. W.; van der Mei, H. C.; Jutte, P. C.; van den Dungen, J. J. A. M.; Schultz, M. J.; van der Laan, B. F. A. M.; Zaat, S. A. J.; Busscher, H. J. Critical Factors in the Translation of Improved Antimicrobial Strategies for Medical Implants and Devices. Biomaterials 2013, 34 (37), 9237-9243.

(37) Costerton, J. W. Bacterial Biofilms: A Common Cause of Persistent Infections. Science 1999, 284 (5418), 1318-1322.

(38) Jaggessar, A.; Shahali, H.; Mathew, A.; Yarlagadda, P. K. D. V. Bio-Mimicking Nano and Micro-Structured Surface Fabrication for Antibacterial Properties in Medical Implants. J. Nanobiotechnol. 2017, 15 (1), 1 DOI: 10.1186/s12951-017-0306-1.

(39) Katsikogianni, M. G.; Wood, D. J.; Missirlis, Y. F. Biomaterial Functionalized Surfaces for Reducing Bacterial Adhesion and Infection. In Handbook of Bioceramics and Biocomposites; Antoniac, I. V., Ed.; Springer International Publishing: Cham, 2015; pp 1-28. DOI: 10.1007/978-3-319-09230-0 32-1.

(40) Nir, S.; Reches, M. Bio-Inspired Antifouling Approaches: The Quest towards Non-Toxic and Non-Biocidal Materials. Curr. Opin. Biotechnol. 2016, 39, 48-55.

(41) Bixler, G. D.; Theiss, A.; Bhushan, B.; Lee, S. C. Anti-Fouling Properties of Microstructured Surfaces Bio-Inspired by Rice Leaves and Butterfly Wings. J. Colloid Interface Sci. 2014, 419, 114-133. 
(42) Magin, C. M.; Cooper, S. P.; Brennan, A. B. Non-Toxic Antifouling Strategies. Mater. Today 2010, 13 (4), 36-44.

(43) Edwards, K. J.; Rutenberg, A. D. Microbial Response to Surface Microtopography: The Role of Metabolism in Localized Mineral Dissolution. Chem. Geol. 2001, 180 (1-4), 19-32.

(44) Anselme, K.; Davidson, P.; Popa, A. M.; Giazzon, M.; Liley, M.; Ploux, L. The Interaction of Cells and Bacteria with Surfaces Structured at the Nanometre Scale. Acta Biomater. 2010, 6 (10), $3824-3846$.

(45) Zhao, N.; Wang, Z.; Cai, C.; Shen, H.; Liang, F.; Wang, D.; Wang, C.; Zhu, T.; Guo, J.; Wang, Y.; Liu, X.; Duan, C.; Wang, H.; Mao, Y.; Jia, X.; Dong, H.; Zhang, X.; Xu, J. Bioinspired Materials: From Low to High Dimensional Structure. Adv. Mater. 2014, 26 (41), 6994-7017.

(46) Damodaran, V. B.; Murthy, N. S. Bio-Inspired Strategies for Designing Antifouling Biomaterials. Biomater. Res. 2016, 20 (1), 1 DOI: 10.1186/s40824-016-0064-4.

(47) Bixler, G. D.; Bhushan, B. Rice and Butterfly Wing Effect Inspired Low Drag and Antifouling Surfaces: A Review. Crit. Rev. Solid State Mater. Sci. 2015, 40 (1), 1-37.

(48) Wu, J.-G.; Chen, J.-H.; Liu, K.-T.; Luo, S.-C. Engineering Antifouling Conducting Polymers for Modern Biomedical Applications. ACS Appl. Mater. Interfaces 2019, 11 (24), 21294-21307.

(49) Mehrjou, B.; Mo, S.; Dehghan-Baniani, D.; Wang, G.; Qasim, A. M.; Chu, P. K. Antibacterial and Cytocompatible Nanoengineered Silk-Based Materials for Orthopedic Implants and Tissue Engineering. ACS Appl. Mater. Interfaces 2019, 11 (35), 31605-31614.

(50) Lou, H.-Y.; Zhao, W.; Li, X.; Duan, L.; Powers, A.; Akamatsu, M.; Santoro, F.; McGuire, A. F.; Cui, Y.; Drubin, D. G.; Cui, B. Membrane Curvature Underlies Actin Reorganization in Response to Nanoscale Surface Topography. Proc. Natl. Acad. Sci. U. S. A. 2019, 116 (46), 23143-23151.

(51) Galeotti, F.; Andicsova, A.; Yunus, S.; Botta, C. Precise Surface Patterning of Silk Fibroin Films by Breath Figures. Soft Matter 2012, 8 (17), 4815-4821.

(52) Galeotti, F.; Hartmann, L.; Botta, C. Robust Surface Patterning by Parylene-Reinforced Breath Figures: An Enabling Tool for Liquid Crystal Microcell Arrays. J. Colloid Interface Sci. 2016, 465, 47-53.

(53) Galeotti, F.; Trespidi, F.; Timò, G.; Pasini, M. Broadband and Crack-Free Antireflection Coatings by Self-Assembled Moth Eye Patterns. ACS Appl. Mater. Interfaces 2014, 6 (8), 5827-5834.

(54) Hu, X.; Shmelev, K.; Sun, L.; Gil, E.-S.; Park, S.-H.; Cebe, P.; Kaplan, D. L. Regulation of Silk Material Structure by TemperatureControlled Water Vapor Annealing. Biomacromolecules 2011, 12 (5), $1686-1696$

(55) Wang, L.-S.; Gupta, A.; Duncan, B.; Ramanathan, R.; Yazdani, M.; Rotello, V. M. Biocidal and Antifouling Chlorinated Protein Films. ACS Biomater. Sci. Eng. 2016, 2 (11), 1862-1866.

(56) Zhang, A.; Bai, H.; Li, L. Breath Figure: A Nature-Inspired Preparation Method for Ordered Porous Films. Chem. Rev. 2015, 115 (18), 9801-9868.

(57) Manabe, K.; Nishizawa, S.; Shiratori, S. Porous Surface Structure Fabricated by Breath Figures That Suppresses Pseudomonas Aeruginosa Biofilm Formation. ACS Appl. Mater. Interfaces 2013, 5 (22), 11900-11905.

(58) Lawrence, B. D.; Omenetto, F.; Chui, K.; Kaplan, D. L. Processing Methods to Control Silk Fibroin Film Biomaterial Features. J. Mater. Sci. 2008, 43 (21), 6967-6985.

(59) Lawrence, B. D.; Pan, Z.; Weber, M. D.; Kaplan, D. L.; Rosenblatt, M. I. Silk Film Culture System for in Vitro Analysis and Biomaterial Design. J. Visualized Exp. 2012, 62, No. e3646.

(60) Meinel, L.; Hofmann, S.; Karageorgiou, V.; Kirker-Head, C.; McCool, J.; Gronowicz, G.; Zichner, L.; Langer, R.; VunjakNovakovic, G.; Kaplan, D. L. The Inflammatory Responses to Silk Films in Vitro and in Vivo. Biomaterials 2005, 26 (2), 147-155.

(61) Yang, Y.; Chen, X.; Ding, F.; Zhang, P.; Liu, J.; Gu, X. Biocompatibility Evaluation of Silk Fibroin with Peripheral Nerve Tissues and Cells in Vitro. Biomaterials 2007, 28 (9), 1643-1652.
(62) Antognazza, M. R.; Di Paolo, M.; Ghezzi, D.; Mete, M.; Di Marco, S.; Maya-Vetencourt, J. F.; Maccarone, R.; Desii, A.; Di Fonzo, F.; Bramini, M.; Russo, A.; Laudato, L.; Donelli, I.; Cilli, M.; Freddi, G.; Pertile, G.; Lanzani, G.; Bisti, S.; Benfenati, F. Characterization of a Polymer-Based, Fully Organic Prosthesis for Implantation into the Subretinal Space of the Rat. Adv. Healthcare Mater. 2016, 5 (17), 2271-2282.

(63) Ding, S.; Kingshott, P.; Thissen, H.; Pera, M.; Wang, P.-Y. Modulation of Human Mesenchymal and Pluripotent Stem Cell Behavior Using Biophysical and Biochemical Cues: A Review. Biotechnol. Bioeng. 2017, 114 (2), 260-280.

(64) Koseoglu, H.; Aslan, G.; Esen, N.; Sen, B. H.; Coban, H. Ultrastructural Stages of Biofilm Development of Escherichia Coli on Urethral Catheters and Effects of Antibiotics on Biofilm Formation. Urology 2006, 68 (5), 942-946.

(65) Bazaka, K.; Crawford, R. J.; Ivanova, E. P. Do Bacteria Differentiate between Degrees of Nanoscale Surface Roughness? Biotechnol. J. 2011, 6 (9), 1103-1114.

(66) Watson, G. S.; Green, D. W.; Schwarzkopf, L.; Li, X.; Cribb, B. W.; Myhra, S.; Watson, J. A. A Gecko Skin Micro/Nano Structure A Low Adhesion, Superhydrophobic, Anti-Wetting, Self-Cleaning, Biocompatible, Antibacterial Surface. Acta Biomater. 2015, 21, 109122.

(67) Nelson, D. E.; Young, K. D. Penicillin Binding Protein 5 Affects Cell Diameter, Contour, and Morphology of Escherichia Coli. J. Bacteriol. 2000, 182 (6), 1714-1721.

(68) Grossman, N.; Ron, E. Z.; Woldringh, C. L. Changes in Cell Dimensions During Amino Acid Starvation of Escherichia Coli. J. Bacteriol. 1982, 152, 7.

(69) Förch, R.; Schönherr, H.; Schonherr, H.; Jenkins, A. T. A. Surface Design: Applications in Bioscience and Nanotechnology; John Wiley \& Sons, 2009.

(70) Wu, X.; Wang, S. Regulating MC3T3-E1 Cells on Deformable Poly $(\varepsilon$-Caprolactone $)$ Honeycomb Films Prepared Using a Surfactant-Free Breath Figure Method in a Water-Miscible Solvent. ACS Appl. Mater. Interfaces 2012, 4 (9), 4966-4975.

(71) Brown, P. S.; Talbot, E. L.; Wood, T. J.; Bain, C. D.; Badyal, J. P. S. Superhydrophobic Hierarchical Honeycomb Surfaces. Langmuir 2012, 28 (38), 13712-13719.

(72) Sykes, E. A.; Albanese, A.; Chan, W. C. W. Implantable Waveguides. Nat. Photonics 2013, 7 (12), 940-941.

(73) Qiao, X.; Qian, Z.; Li, J.; Sun, H.; Han, Y.; Xia, X.; Zhou, J.; Wang, C.; Wang, Y.; Wang, C. Synthetic Engineering of Spider Silk Fiber as Implantable Optical Waveguides for Low-Loss Light Guiding. ACS Appl. Mater. Interfaces 2017, 9 (17), 14665-14676.

(74) Huby, N.; Vié, V.; Renault, A.; Beaufils, S.; Lefèvre, T.; PaquetMercier, F.; Pézolet, M.; Bêche, B. Native Spider Silk as a Biological Optical Fiber. Appl. Phys. Lett. 2013, 102 (12), 123702.

(75) Lawrence, B. D.; Cronin-Golomb, M.; Georgakoudi, I.; Kaplan, D. L.; Omenetto, F. G. Bioactive Silk Protein Biomaterial Systems for Optical Devices. Biomacromolecules 2008, 9 (4), 1214-1220.

(76) Tao, H.; Siebert, S. M.; Pritchard, E. M.; Sassaroli, A.; Panilaitis, B.; Brenckle, M. A.; Amsden, J. J.; Levitt, J. M.; Fantini, S.; Kaplan, D. L.; Omenetto, F. G. Implantable Resorbable Micro-Prism Arrays for Real-Time Drug Release Monitoring. In Conference on Lasers and Electro-Optics 2012; OSA: San Jose, CA, 2012; p CTh4L.8. DOI: 10.1364/CLEO SI.2012.CTh4L.8.

(77) Omenetto, F. G.; Kaplan, D. L. A New Route for Silk. Nat. Photonics 2008, 2 (11), 641-643.

(78) Maya-Vetencourt, J. F.; Ghezzi, D.; Antognazza, M. R.; Colombo, E.; Mete, M.; Feyen, P.; Desii, A.; Buschiazzo, A.; Di Paolo, M.; Di Marco, S.; Ticconi, F.; Emionite, L.; Shmal, D.; Marini, C.; Donelli, I.; Freddi, G.; Maccarone, R.; Bisti, S.; Sambuceti, G.; Pertile, G.; Lanzani, G.; Benfenati, F. A Fully Organic Retinal Prosthesis Restores Vision in a Rat Model of Degenerative Blindness. Nat. Mater. 2017, 16 (6), 681-689.

(79) Ghezzi, D.; Antognazza, M. R.; Maccarone, R.; Bellani, S.; Lanzarini, E.; Martino, N.; Mete, M.; Pertile, G.; Bisti, S.; Lanzani, G.; 
Benfenati, F. A Polymer Optoelectronic Interface Restores Light Sensitivity in Blind Rat Retinas. Nat. Photonics 2013, 7 (5), 400-406. (80) Tullii, G.; Desii, A.; Bossio, C.; Bellani, S.; Colombo, M.; Martino, N.; Antognazza, M. R.; Lanzani, G. Bimodal Functioning of a Mesoporous, Light Sensitive Polymer/Electrolyte Interface. Org. Electron. 2017, 46, 88-98.

(81) Vaquero, S.; Bossio, C.; Bellani, S.; Martino, N.; Zucchetti, E.; Lanzani, G.; Antognazza, M. R. Conjugated Polymers for the Optical Control of the Electrical Activity of Living Cells. J. Mater. Chem. B 2016, 4 (31), 5272-5283. 